\title{
Criterios para la selección de dispositivos inteligentes en tenis
}

\author{
Ángel Iván Fernández-García y Gema Torres-Luque
}

\author{
Universidad de Jaén, Andalusia, España
}

RESUMEN

Existen mayores posibilidades para los entrenadores de dispositivos que contribuyen al proceso de entrenamiento del tenis. Sin embargo, en muchas ocasiones la elección de uno $u$ otro instrumento genera incertidumbre debido a la variedad existente en el mercado y al desconocimiento que hay sobre el rendimiento de los diferentes aparatos. Por ello, el objetivo del presente artículo es el de presentar una información técnica detallada sobre el rendimiento de los instrumentos que aportan datos técnicos y cinemáticos y elaborar unos criterios en los que puedan basarse entrenadores y jugadores para adquirir el más apropiado a sus necesidades.

Palabras clave: : tenis, nuevas

\author{
Palabras clave: : tenis, nuevas \\ Recibido: 27 Abril 2018 \\ Aceptado: 10 Junio 2018 \\ Autor correspondiente: \\ Ángel Iván Fernández \\ Universidad de Jaén, \\ Andalusia, España. Correo \\ electrónico: \\ angelivanfg@hotmail.com, \\ gtluque@ujaen.es
}

\section{INTRODUCCIÓN}

En el proceso de desarrollo de un jugador de tenis, un elemento clave a la hora de programar las actuaciones del técnico en el proceso de enseñanza-aprendizaje lo constituye el análisis y la evaluación de las diferentes áreas de trabajo (Sanz, 2012). Gracias a los avances de la ciencia, la información de la que pueden disponer los entrenadores es mucho más potente, ya que los datos son más completos y precisos, además de que se presentan de manera inmediata y fácilmente interpretable.

En este sentido, la evaluación y el análisis de la técnica y la cinemática ha sido uno de los aspectos más sistematizados en el tenis, por delante de otras áreas como la táctica o la psicológica. Las primeras referencias en cuanto al uso de la tecnología para el desarrollo de jugadores de tenis data de comienzos del siglo XX (Beldam y Vaile, 1905; Vaile, 1906; Paret, 1926; Lacoste, 1928). Sin embargo, no ha sido hasta hace poco que la tecnología ha aumentado exponencialmente en el mercado, ofreciendo un proceso de obtención de datos accesible y económico, lo que está supliendo la técnica del "ojo clínico del entrenador", sistema que presenta deficiencias en cuanto a la precisión de los datos basado en exceso en la subjetividad (Sanz, 2012).

Además, entre los beneficios del uso de las nuevas herramientas, es la mayor motivación que supone su aplicación en el proceso de enseñanza-aprendizaje para jugadores y entrenadores, ya que aportan evidencias del nivel técnico en tiempo real y permiten comprobar los progresos comparando diferentes sesiones de entrenamiento o partidos. Además, estos datos pueden compartirse por diferentes plataformas sociales (Quinlan, 2013).

El objetivo del presente trabajo es analizar el rendimiento de las herramientas que ofrecen información técnica y cinemática de la acción de la raqueta sobre la pelota y exponer los criterios en los que deben basarse entrenadores y jugadores a la hora de su elección.

\section{MÉTODOS Y PROCEDIMIENTOS}

Los aparatos analizados fueron: Sony Smart Tennis Sensor, Babolat Pop, Babolat Play (Pure Drive), Zepp Tennis, Zepp Tennis 2 y Persona Coach de Artengo. Se llevó a cabo un análisis detallado de las páginas web oficiales de cada uno de los dispositivos seleccionados con el propósito de conocer el rendimiento que ofrece cada uno de ellos:

\section{FUNCIONALIDAD Y PROCEDIMIENTOS}

A continuación, se presenta una clasificación de los diferentes dispositivos según su capacidad de registro en distintos ítems. De hecho, la tabla 1, muestra la capacidad de registrar aspectos relacionados con el volumen de entrenamiento en los dispositivos seleccionados. 


\begin{tabular}{|c|c|c|c|c|c|c|}
\hline & $\begin{array}{l}\text { Persond } \\
\text { Cosch } \\
\text { Artentos }\end{array}$ & Bubolat & $\begin{array}{l}\text { Babolat } \\
\text { play }\end{array}$ & $\begin{array}{l}\text { Sonvy } \\
\text { Smart } \\
\text { Tennis } \\
\text { sentor }\end{array}$ & $\begin{array}{l}26 s p \\
\text { Tennis }\end{array}$ & $\frac{\operatorname{laph}_{2}}{2}$ \\
\hline 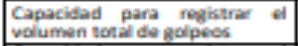 & $r$ & $\checkmark$ & $r$ & 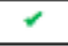 & 2 & 7 \\
\hline 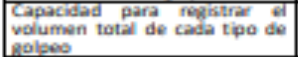 & $\checkmark$ & $\checkmark$ & 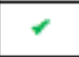 & $\checkmark$ & $\checkmark$ & $r$ \\
\hline 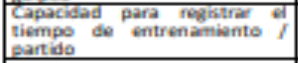 & * & $\checkmark$ & $r$ & $\checkmark$ & $\checkmark$ & $\gamma$ \\
\hline $\begin{array}{l}\text { Frecuencia de golpeos por } \\
\text { minuse }\end{array}$ & $\approx$ & $r$ & $r$ & $=$ & $\approx$ & $\mathrm{x}$ \\
\hline 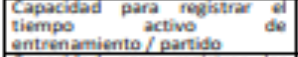 & $\mathbf{x}$ & * & 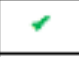 & $x$ & ' & $\gamma$ \\
\hline 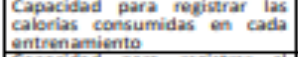 & × & $\checkmark$ & ' & $\checkmark$ & $\checkmark$ & $\gamma$ \\
\hline 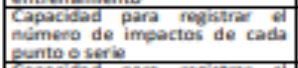 & x & * & x & $x$ & x & × \\
\hline 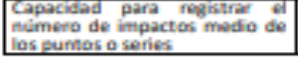 & x & x & x & $x$ & x & 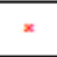 \\
\hline
\end{tabular}

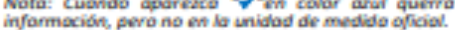

Tabla 1. Capacidad para registrar aspectos relacionados con el volumen de entrenamienta.

En la tabla 2, se muestra la capacidad de los dispositivos en cuanto a aspectos relacionados con el golpeo.

\begin{tabular}{|c|c|c|c|c|c|c|}
\hline & $\begin{array}{c}\text { Perional } \\
\text { Coich } \\
\text { Antenglo } \\
\end{array}$ & Babolat & $\begin{array}{l}\text { Babolin } \\
\text { Pley }\end{array}$ & \begin{tabular}{|l} 
Somin \\
Smant \\
Tennis \\
Sensert
\end{tabular} & $\begin{array}{l}\text { Zepp } \\
\text { Ternis }\end{array}$ & $\begin{array}{c}2 e p e \\
\text { Fins } \\
2 \\
\end{array}$ \\
\hline 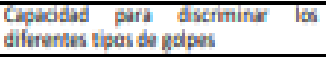 & 7 & 7 & 7 & 7 & 7 & $\gamma$ \\
\hline 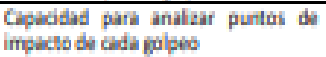 & $x$ & $x$ & $\mathrm{x}$ & $\checkmark$ & $\mathrm{x}$ & $\checkmark$ \\
\hline 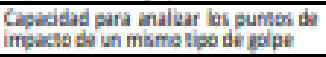 & 7 & $x$ & 7 & 7 & 7 & 1 \\
\hline $\begin{array}{l}\text { Copedidad part dicciminar entre } \\
\text { afecto iftado y contado }\end{array}$ & $x$ & 7 & $\checkmark$ & 7 & 7 & 1 \\
\hline 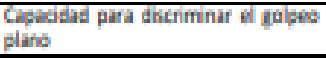 & $\mathrm{x}$ & $x$ & $\bar{\gamma}$ & $x$ & $\bar{\gamma}$ & 7 \\
\hline 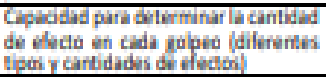 & $x$ & 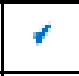 & $\checkmark$ & 7 & 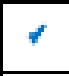 & $\downarrow$ \\
\hline 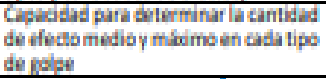 & $x$ & $\gamma$ & $\checkmark$ & 1 & $\checkmark$ & $\checkmark$ \\
\hline
\end{tabular}

pere no ent lo unidad de midide officiat

Tabla 2. Capocidad para discriminar los diferentes raquetas, tipos de golpes, efectos y analizar los puntos de impacto.

En la tabla 3, destaca los aspectos relacionados con la velocidad.

\begin{tabular}{|c|c|c|c|c|c|c|}
\hline & $\begin{array}{l}\text { Perianal } \\
\text { Coach } \\
\text { Artentyo }\end{array}$ & Bindat & $\begin{array}{l}\text { Babolat } \\
\text { Play }\end{array}$ & $\begin{array}{l}\text { Sany } \\
\text { Smart } \\
\text { Tennis } \\
\text { Semar }\end{array}$ & $\begin{array}{l}\text { Zepp } \\
\text { Tennis }\end{array}$ & $\begin{array}{l}\text { Zupe } \\
\operatorname{Tenin} \\
2\end{array}$ \\
\hline $\begin{array}{l}\text { Capacidad pura seterminar la vilocisad de } \\
\text { la pelota en casa golpeo }\end{array}$ & $x$ & $x$ & $x$ & 1 & 7 & 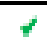 \\
\hline 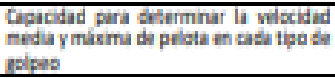 & $\mathrm{K}^{*}$ & 1 & $\checkmark$ & 1 & $\downarrow$ & $\gamma$ \\
\hline $\begin{array}{l}\text { Capacidad pura deferminar la vilocitad do } \\
\text { caba wwing }\end{array}$ & $x$ & $x$ & $x$ & 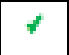 & 7 & $x$ \\
\hline 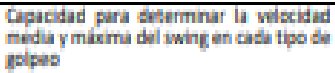 & $x$ & $x$ & $x$ & 7 & $x$ & $x$ \\
\hline $\begin{array}{l}\text { Copacidad para beterminar la cantidad de } \\
\text { effecto en cada golpeo (velocidas) }\end{array}$ & $x$ & $x$ & K & 1 & $x$ & 1 \\
\hline 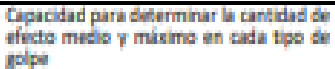 & $x$ & 1 & 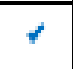 & 1 & $\downarrow$ & $\gamma$ \\
\hline
\end{tabular}

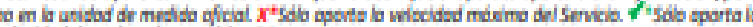

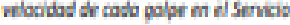

Tabla 3. Capacidad para anollzar la velocidad de pelota y del swing y la cantidod de efecto.
En la tabla 4, se muestran las variables relacionadas con las estadística de juego.

\begin{tabular}{|c|c|c|c|c|c|c|}
\hline & $\begin{array}{c}\text { Personal } \\
\text { Coach } \\
\text { Artenge }\end{array}$ & \begin{tabular}{|c|} 
Inabolat \\
Pop
\end{tabular} & $\begin{array}{l}\text { Babsiat } \\
\text { Divy }\end{array}$ & $\begin{array}{l}\text { Sorvy } \\
\text { Smart } \\
\text { Terni } \\
\text { Senvar }\end{array}$ & Tenpp & $\begin{array}{c}\text { Zepp } \\
\text { Tent } \\
2\end{array}$ \\
\hline 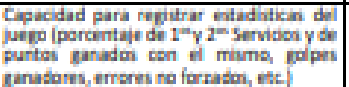 & x & $x$ & $x$ & $x$ & x & $\gamma$ \\
\hline 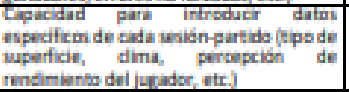 & x & $\gamma$ & $\downarrow$ & 1 & x & $x$ \\
\hline 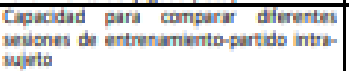 & x & $\gamma$ & 7 & 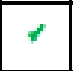 & 7 & 1 \\
\hline $\begin{array}{l}\text { Copacdad para comparir rendimientai } \\
\text { inter-ujytos }\end{array}$ & $x$ & 7 & 7 & 7 & 7 & 7 \\
\hline $\begin{array}{l}\text { Cipacidad para afferenciar entre } \\
\text { entrenamientas ypartidas }\end{array}$ & x & $\checkmark$ & $\checkmark$ & x & $x$ & $\gamma$ \\
\hline $\begin{array}{l}\text { Ciepacidad pura regotrar el rinultado de un } \\
\text { partido }\end{array}$ & $\mathrm{x}$ & 7 & 7 & $x$ & $\mathrm{x}$ & 7 \\
\hline 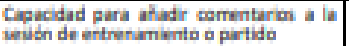 & x & $x$ & $x$ & $\gamma$ & $x$ & $x$ \\
\hline
\end{tabular}

Tabla 4. Capacidad para registrar aspectos estadísticos del juego y reallzar comparaciones intra e inter-sujeta.

En la tabla 5, se observan las posibilidades en cuanto a la generación de vídeos y la obtención de datos.

\begin{tabular}{|c|c|c|c|c|c|c|}
\hline & $\begin{array}{l}\text { Pernonal } \\
\text { Couch } \\
\text { Arteryge }\end{array}$ & Babolat & $\begin{array}{l}\text { Babelat } \\
\text { Play }\end{array}$ & \begin{tabular}{|l|} 
Sany \\
Smit \\
Terni \\
Sinigr \\
\end{tabular} & Tepp & $\begin{array}{c}2 \text { epp } \\
\text { Tenis } \\
2\end{array}$ \\
\hline Capucisad para regatre videos & $x$ & $x$ & $x$ & 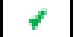 & $\checkmark$ & $\downarrow$ \\
\hline $\begin{array}{l}\text { Capucisad para visulitar la videas a } \\
\text { cimura lenta }\end{array}$ & $x$ & $\mathrm{x}$ & $x$ & $\boldsymbol{\gamma}$ & $\checkmark$ & $\downarrow$ \\
\hline 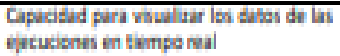 & $x$ & $\mathrm{x}$ & $x$ & 1 & 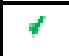 & $\downarrow$ \\
\hline 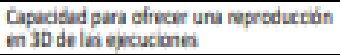 & $x$ & $\mathrm{x}$ & $x$ & $x$ & $\Delta$ & $\checkmark$ \\
\hline 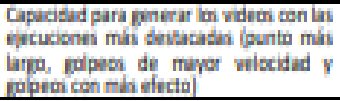 & $x$ & $\mathrm{x}$ & $x$ & $\mathrm{x}$ & $\mathrm{x}$ & $\downarrow$ \\
\hline 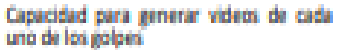 & $\mathrm{x}$ & $\mathrm{x}$ & $x$ & $\boldsymbol{\gamma}$ & $x$ & $\checkmark$ \\
\hline
\end{tabular}

\section{Tabla 5. Copocidad para registrar videos de las ejecuciones y aportar datos al instante.}

Criterios a la hora de seleccionar los aparatos

A continuación, se exponen algunas de las razones en las que deben basarse entrenadores y jugadores para realizar la selección del aparato más adecuado a sus necesidades:

a) Desde el punto de vista del entrenador:

- Cantidad de alumnos con los que trabajen: para el caso de entrenadores de escuela que trabajen con un volumen amplio de alumnos lo ideal será buscar un dispositivo que pueda ser utilizado con la mayor cantidad de marcas y modelos posibles de raqueta. Por su parte, los entrenadores a título individual o de un grupo reducido, sí que podrían plantearse la opción de elegir algún modelo algo más restringido. 


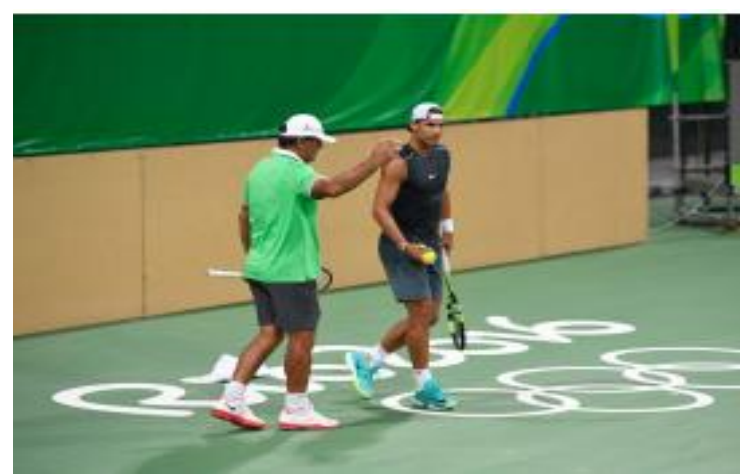

- Nivel de información requerida según el nivel de los alumnos y los conocimientos del entrenador (competición versus aficionados): los entrenadores deberán valorar el tipo y cantidad de información que sean capaces de interpretar y que además necesiten para mejorar el nivel de los alumnos con los que trabajen. A medida que el nivel de los alumnos y el conocimiento de los técnicos es mayor, los requerimientos de información también son más elevados. Un posible ejemplo de ello podrían ser los datos relacionados con la cantidad de efectos, la carga de entrenamiento o la comparativa de rendimientos entre diferentes sesiones de entrenamiento o partidos.

- Realizar grabaciones: en el caso de que quiera utilizarse la opción "video" con datos específicos sobre las ejecuciones en tiempo real, habrá que decantarse por las herramientas Sony Smart Tennis Sensor y los dos modelos de la marca Zepp, ya que son las únicas que disponen de esta posibilidad.

- Datos estadísticos de competición: para entrenadores que necesiten obtener datos estadísticos del juego a la vez que los técnicos y cinemáticos, el único dispositivo que ofrece esta posibilidad es el sensor Zepp 2.

- Tipo de población: una de las grandes limitaciones para la enseñanza del tenis a edades tempranas, es que los dispositivos no están adaptados para raquetas más pequeñas, con lo que quedan excluido su uso para raquetas junior hasta las más pequeñas de pre-tenis.

b) Desde el punto de vista del jugador para uso individual:

- Frecuencia con la que rompen el cordaje: si es un jugador que rompe cuerdas de manera habitual o alterna diferentes raquetas, deberá elegir un sensor externo que le permita cambiarlo de raqueta cada vez que lo necesite, mientras que si se trata de un jugador aficionado que apenas la cambia, podrá optar por uno interno.

- Compartir datos y rendimiento en plataformas sociales: en el caso de que los jugadores quieran comparar sus resultados con otros usuarios que utilicen la misma herramienta, podrán adquirir todas ellas, salvo el Personal Coach de Artengo.

- Si el jugador practica o no con entrenador: en el caso de que un jugador pueda contar con la ayuda de un entrenador, deberá en primer lugar pedirle consejo a él, sobre cuál es la herramienta más adecuada según sus características. Tal y como se ha comentado anteriormente, a medida que el nivel del jugador y los conocimientos del técnico son más elevados, los requerimientos de información, también serán mayores.

\section{CONCLUSIÓN}

El uso de los dispositivos inteligentes en tenis, son sin lugar a dudas, una ayuda considerable al proceso de entrenamiento, teniendo en cuenta que están para contribuir y ayudar, pero nunca para sustituir al entrenador. La elección de uno u otro dispositivo, dependerá fundamentalmente de aquellos parámetros que se quieran controlar, si van más dirigidos hacia el conocimiento de la dinámica de golpeo, tener vídeos inmediatos, conseguir estadísticas de competición, etc. Sin duda, las tablas que se presentan ayuden a una elección más acertada en función del interés concreto.

\section{REFERENCIAS}

Beldam G. \& Vaile, P.A. (1905). Great lawn tennis players. Their methods illustrated. Illustrated by 229 action-photograpfs. Londres: Mac Millan \& Co.

Lacoste, R. (1928). Tennis. Paris: Grasset.

Quinlan, G. (2013). El uso de las aplicaciones para mejorar el entrenamiento: La aplicación técnica de Tenis Australia. ITF Coaching \& Sport Science Review. 59, 22-24

Sanz, D., y Terroba, A.. (2012). Aplicación de las nuevas tecnologías al análisis de la táctica en el tenis. ITF Coaching \& Sport Science Review. 20 (56), 23-25.

Vaile, P.A. (1906). The strokes and Science of lawn tennis, New York: American Sports Publishing Company.

\section{CONTENIDO ITF ACADEMY RECOMENDADO (HAZ CLICK ABAJO)}

\section{ITF Academy}

Derechos de Autor (c) 2018 Ángel Iván Fernández-García y Gema Torres-Luque

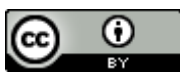

Este texto está protegido por una licencia CreativeCommons 4.0.

Usted es libre para Compartir —copiar y redistribuir el material en cualquier medio o formato- y Adaptar el documento - remezclar, transformar y crear a partir del material- para cualquier propósito, , incluso para fines comerciales, siempre que cumpla la condición de:

Atribución: Usted debe dar crédito a la obra original de manera adecuada, proporcionar un enlace a la licencia, e indicar si se han realizado cambios. Puede hacerlo en cualquier forma razonable, pero no de forma tal que sugiera que tiene el apoyo del licenciante o lo recibe por el uso que hace de la obra.

Resumendelicencia - Textocompletodelalicencia 